\title{
Model Reduction of Power System by Modified Balanced Truncation Method
}

\author{
Santosh Kumar Suman, Awadhesh Kumar" \\ Department of Electrical Engineering, Madan Mohan Malaviya University of Technology, Gorakhpur, Uttar Pradesh, India
}

Received July 4, 2020; Revised October 13, 2020; Accepted October 24, 2020

\section{Cite This Paper in the following Citation Styles}

(a): [1] Santosh Kumar Suman, Awadhesh Kumar, "Model Reduction of Power System by Modified Balanced Truncation Method," Universal Journal of Control and Automation, Vol. 8, No. 3, pp. 41 - 52, 2020. DOI: 10.13189/ujca.2020.080301.

(b): Santosh Kumar Suman, Awadhesh Kumar (2020). Model Reduction of Power System by Modified Balanced Truncation Method. Universal Journal of Control and Automation, 8(3), 41 - 52. DOI: 10.13189/ujca.2020.080301.

Copyright $\bigcirc 2020$ by authors, all rights reserved. Authors agree that this article remains permanently open access under the terms of the Creative Commons Attribution License 4.0 International License

\begin{abstract}
In this article, we are exploring and implementing the new model order reduction (MOR) method for Large-Scale Linear Dynamic System (LSLDS) to achieve reduced order. These technologies are designed to better understand and explain LSLDS based on the Modified Balanced Truncation Method (BTM). This refers to continuous/discrete LTI structures that are minimal / non-minimum. This reduced method allows MOR to preserve complete parameters with reasonable accuracy. The approach is based on the maintenance of dominant system modes and a relatively small state truncation. As the reduced-order model (ROM) is derived from the retention of dominant modes, the reduction remains stable. The main demerit of the balanced truncation method is that the ROM, stable states, does not match the original structures. By modified BTM to narrow the deviations in the ROM transfer function matrix, a gain factor is added to adjust the steady-state values of the reduction system without altering the dynamic behaviour of the system. The proposed method has been successfully applied to a real-time single area power system with ease of extension to a discrete-time case and the results obtained show the efficacy of the method. Application model and the results obtained indicate the effectiveness of the methodology. The time response of the system has been demonstrated by the proposed method, which proves to be excellent match, effectiveness and superiority compared to the response of other approaches in the literature review of the original system.
\end{abstract}

Keywords Model Order Reduction, Power System, Gain Factor, Balanced Truncation Method, Steady State

\section{Introduction}

Electric power technology is emerging as a result of electricity demand. As the most demanding artificial device, the entire power system consists of many

processes, such as output, transmission and distribution. Systems are built in different areas, typically linked by transmission lines or linkages to tie-lines, painless areas [1][2]. This causes the system model to have an extremely high order. Both regional frequency and tie-line exchange variations in such interconnected power systems networks are often induced by unforeseen demand only for power loads, uncertain system parameters, modelling error and disturbances due to various environmental conditions[3][4]. Thus, during transient disturbances such as faults, line travel or any overload, the reliability of the power system is necessary to maintain the specified synchronism and voltage levels[5][6][7]. It is therefore capable of minimising the model of the power system so that simulation and controller design can be simplified [8].

Electrical energy technology is the biggest problem in all respects concerning the dynamic behaviour of a larger system [9][10][11]. All these complex and large systems with traditional techniques are difficult to model. The hybrid system has also been shown to be large (extensive) if it is intended for computational evaluation and practical reasons in different coordinated systems or small systems [12][13][14]. If not, the system is large and so large that conventional modelling, analysis, System design and 
computing approaches cannot provide the appropriate solutions with reasonable realistic computing activities [15][16][17]. The study of such a physical device begins with a structured model that can be considered an enthusiast example. The control objective encourages the development and evaluation of a model [16][18][19]. In the first part, high-level negotiations on new technologies and the basic rules for a dense model system are considered both in view and in the industry[13][20]. Mostly in two ways, multiple MOR approaches have been given to frequency and time domains [21]. Researchers' methods of reduction have many advantages and drawbacks to them. A common flaw in the method is that even if the Higher-Order System (HOS) is stable, the reduced-order system is unstable [22][11][23]. Moreover, most of the time, the steady-state matching of the original system with its lower order representation fails. Additional drawbacks are poor accuracy in normal high-frequency ranges that may have non-minimum phase attributes [24][25][26] [27]. There are several methods available even more in the literature to the frequency and time-domain model of HOS such as a ROM algorithm using Pade Approximation [28][29][30][31][32][16][33]. MOR of state LTI systems based on the theory of balanced realization was firstly recommended by [34] in which the balanced realization term has been chosen to derive a balance between the controllability and observability of states in its system configuration[35]. The balanced truncated (BT) reduced model achieved by a balanced realized model following the truncation of less controllable and less observable states. It has been found that the model thus identified does not retain the DC gain or the steady-state of the actual (original) system[36]. Poor removal of the subsystem was introduced to maintain the balanced truncation benefit of DC gain with SPA [37][38][39][40][41][42][43][44]. The preservation of DC BT minimal system gains has been defined through a singular perturbation approach that is applicable when the system to be reduced is stable, minimal and internally balanced [45][44]. In this, the investigator suggested a modified BT approach applicable to LSLDS with outstanding DC gain matching [46]. The benefit of the approach lies not only to the matching of steady state but its applicability continuous to discrete time system as well, which has been confirmed through examples taken from literature.

\section{Methodology for Rom}

\subsection{Problem Statement}

Find the following State Space matrix n represented by a SISO system.

\subsubsection{Brief description}

This brief discussion focuses on computing the low-order approximation estimation of linear dynamic systems.

Consider the system of continuous-time-LTI SISO state-space as specified

$$
\sum:\left\{\begin{array}{l}
\dot{x}(t)=A x(t)+B u(t) \\
y(t)=C x(t)+D u(t)
\end{array} \Leftrightarrow \sum:=\left(\begin{array}{l:l}
A & B \\
\hdashline C & D
\end{array}\right)\right.
$$

where $x \in \square^{n}$ is n-dimensional of the system state(1), $y \in \square^{p}$ is the system output of $\sum, \mathrm{u}(\mathrm{t})$ is the input vector, respectively, $\mathrm{A} \in \square^{n \times n}, \mathrm{~B} \in \square^{n \times m}, \mathrm{C} \in \square^{p \times n}$ and $D \in \square^{p \times m} . x \in \square^{n}$ are state variables.

where $\mathrm{n}$ is a large-value system order, which is a high-dimensional system, with $\mathrm{n}$ extending from several tens/hundreds to thousands as available in large-scale dynamic system control systems [47][48][34][14].

Consider the transfer function of the n-dimensional SISO Dynamical system [49]. Equation (1) is in the form of a transfer function.

$$
\begin{gathered}
G(s)=C\left[s I_{n}-A\right]^{-1} B+D \\
G(s)=\frac{N(s)}{D(s)}=\frac{n_{0}+n_{1} s+n_{2} s^{2}+\cdots n_{m-1} s^{m-1}}{d_{0}+d_{1} s+d_{2} s^{2}+\cdots+d_{n} s^{n}} \\
\text { or } G(s)=\frac{\sum_{i=0}^{m-1} n_{i} s^{i}}{\sum_{i=0}^{n} d_{i} s^{i}}
\end{gathered}
$$

Where $\mathrm{m}$ is less than $\mathrm{n}$ and numerator polynomial $\left(n_{i}\right)$ are constants coefficient and denominator polynomial $\left(d_{i}\right)$ of the original system, correspondingly it is accordingly assumed that the state of the system is such as all the roots lie in the left half of the s-map.

The gain factor of the Original system is $k$

$$
k=\frac{n_{0}}{d_{0}}
$$

The finding ROM $G_{r}(s)$, which in some way has approximated the original system $(G(s))$ and preserves essential parameters of the original system. Such a solution corresponds as closely as possible to the solution of the system for a similar form of input. The ROM is given by

$$
\sum:\left\{\begin{array}{l}
\dot{x}_{r}(t)=A_{r} x_{r}(t)+B_{r} u_{r}(t) \\
y_{r}(t)=C_{r} x_{r}(t)+D_{r} u_{r}(t)
\end{array} \Leftrightarrow \sum_{r}:=\left(\begin{array}{l:l}
A_{r} & B_{r} \\
\hline C_{r} & D_{r}
\end{array}\right)\right.
$$

Where $r, r$ is less than $n$, so that the transfer function of the HOS [50]. The transfer function $G_{r}(s)$ of the reduced model is defined by

$$
\begin{gathered}
G_{r}(s)=C_{r}\left(s I_{r}-A_{r}\right)^{-1} B_{r}+D_{r} \\
G_{r}(s)=\frac{M_{r}(s)}{P_{r}(s)}=\frac{m_{0}+m_{1} s+m_{2} s^{2}+\cdots+m_{r-1} s^{r-1}}{p_{0}+p_{1} s+p_{2} s^{2}+\cdots p_{r-1} s^{r-1}+p_{r} s^{r}}
\end{gathered}
$$


The gain factor of reduced model order is $k_{r}$

$$
\begin{gathered}
k_{r}=\frac{m_{0}}{p_{0}} \\
\text { Or } R_{r}(s)=\frac{\sum_{j=0}^{r-1} m_{j} s^{j}}{\sum_{j=0}^{r} p_{j} s^{j}}
\end{gathered}
$$

Where $m_{j}, n_{j}$ are the unknown parameters scalar constant of the ROM. These parameters are to be obtained while the reduction of a HOS to ROM [51].

\section{Balanced Truncation Method}

The BR method Realization with the controllability grammian (CG) and observability grammian (OG) equal to a diagonal matrix $\sum$ are essential mechanisms for the balancing and adaptation of the composite system[16]. There exists a transformation (T) such that transformed CG and $O G$ is equal to a diagonal matrix. Such as a realization is called a balanced realization or inside balanced realization. Internal balancing of a given realization is the first step into a class of methods for MOR, called balanced truncation method (BTM)[32].

\subsection{The Reduction Algorithm Steps Using the BT Approach is Given Below}

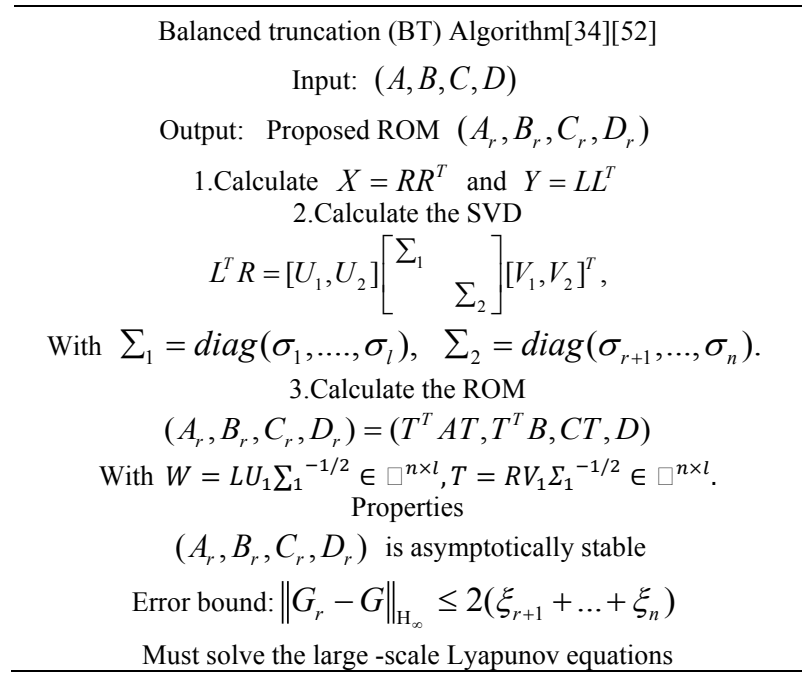

Now the system is balanced which is partitioned as[53][14][48][54].

$$
\begin{aligned}
G_{B a l}(s) & =\left[\begin{array}{c:c}
T A T^{-1} & T^{T} B \\
\hline C T & D
\end{array}\right] \\
& =\left[\begin{array}{c:c}
A_{B a l} & B_{B a l} \\
\hline C_{B a l} & D_{B a l}
\end{array}\right]
\end{aligned}
$$

Balanced which is partitioned as strong subsystem and weak subsystem

$$
=\underbrace{\left[\begin{array}{cc}
A_{11} & B_{1} \\
C_{1} & D_{r}
\end{array}\right]}_{\text {Strong }}+\underbrace{\left[\begin{array}{cc}
A_{22} & B_{2} \\
C_{2} & 0
\end{array}\right]}_{\text {Weak }} \Leftrightarrow \sum:\left[\begin{array}{cc}
\sum_{1} & 0 \\
0 & \Sigma_{2}
\end{array}\right]
$$

Where

$A_{11}$ and $\sum_{1}$ are reduced matrix $(r<n)$

It is call direct-reduction' (DR) reduction of the balanced system. Several well-known outcomes that are related to approximation are offered [38].

\section{A Modified Balanced Truncation Method}

The ROM (6) may be closer (approximately) to the HOS (1) well in the high-frequency band, but generally, it may not do well in the steady-state output deviation between the ROM and the HOS [46].To bring the ROM into line with the original system, we need to change the conventional BTM in that section [23].

Here considers only the single-input single-output (SISO) linear system, in this step only taken an as the transfer function of both systems. Let $G(s)$ and $G_{r}(s)=R_{r}(s)$ be the transfer function of the system (1) and reduced model (6). To reconcile the steady-state value of the ROM, the gain factor is introduced to the ROM[46]. Gain factor $\mathrm{K}$ as follow as

$$
\begin{gathered}
K=\left.\frac{G(s)}{R_{r}(s)}\right|_{s=0} \\
K=\frac{k}{k_{r}}=\frac{\frac{n_{0}}{d_{0}}}{\frac{m_{0}}{p_{0}}}=\frac{n_{0}}{d_{0}} \times \frac{p_{0}}{m_{0}}
\end{gathered}
$$

At this point, $n_{0}$ and $m_{0}$ are positive, $d_{0}$ and $p_{0}$ are nonzero, because the system is controllable, observable, and stable[44]. Then the transfer function of the reduced model becomes the following.

$$
\hat{G}_{r}(s)=K G_{r}(s)=K R_{r}(s)
$$

Steady-state differences may be reduced by utilizing the gain factor, but the dynamic behaviour of the reduced model cannot be consistent with that of the original system[55].

Depending on the final value theorem, the final output of the reduced model can be calculated as the final output of the specified step input.

$$
\lim _{t \rightarrow \infty} y(t)=\lim _{s \rightarrow \infty}\left(s \cdot G_{r}(s) \cdot \frac{1}{s}\right)=\frac{m_{0}}{p_{0}}
$$

It is well known from equation (17) that only the constant terms of the numerator polynomial and denominator polynomial are associated with the steady-state value of $y$. To ensure the stability of the ROM, 
the constant term $p_{0}$ of the denominator polynomial must not be changed. For this reason, we propose that the gain factor $K$ should be included in front of the constant term $m_{0}$ of the numerator polynomial. In this way, the transfer function of the reduced model is modified as follows

$$
\hat{G}_{r}(s)=\frac{\hat{M}_{r}(s)}{P_{r}(s)}=\frac{K\left(m_{0}+m_{1} s+m_{2} s^{2}+\cdots+m_{r-1} s^{r-1}\right)}{p_{0}+p_{1} s+p_{2} s^{2}+\cdots p_{r-1} s^{r-1}+p_{r} s^{r}}
$$

From (18), we can calculate the final output of the reduced model $\hat{G}_{r}(s)$ as follows

$$
\lim _{t \rightarrow \infty} y(t)=\lim _{s \rightarrow \infty}\left(s \cdot \hat{G}_{r}(s) \cdot \frac{1}{s}\right)=\frac{K m_{0}}{p_{0}}=\frac{n_{0}}{d_{0}}
$$

The steady-state output of the reduced model is equal to that of the original systems.

This Modified Balanced Truncation Method can also be extended to a continuous/discrete-time MIMO system to a reduced model for a large-scale dynamic system [46].

\subsection{Discrete-time Stable System by Extended Modified Balanced Truncation Method}

Many Approaches in the continuous and discrete-time system to run in parallel [56] suggested Modified Balanced Truncation Method[46] is also extended to the reduced discrete system by converting the discrete-time system into an equivalent continuous-time system using bilinear mapping and by then reducing it. The reduced system is retransforming to the discrete system using reversing bilinear transformation without losing behaviour. In the last section, we have shown that Modified balance Truncation of a balanced system is fully compatible with Moore's [57] direct truncation method in the continuous-time case. We must now examine whether this is still true for the discrete-time system to do so, we must first determine some of the bilinear mapping characteristics between the complex s-plane and the Z-plane.

We begin with a minimal and stable system with a transfer function, continuous-time, linear, time-invariant (LTI)

$$
G(s)=C\left[s I_{n}-A\right]^{-1} B+D
$$

Assumes that discrete-time, LTI, minimal and stable system

$$
G(z)=H\left(Z I_{n}-\Phi\right)^{-1} G+J
$$

Via the following bilinear transformation, it is obtained from the continuous-time system [14]. The bilinear transformation is characterised by

$$
s=\frac{z-1}{z+1} \Leftrightarrow\left(\text { inverse }: z=\frac{1+s}{1-s}\right)
$$

Therefore, the matrices

$$
\Sigma_{C}=\left[\begin{array}{c:c}
A & B \\
\hdashline C & D
\end{array}\right], \Sigma_{D}=\left[\begin{array}{c:c}
F & G \\
\hdashline H & J
\end{array}\right]
$$

Which maps the left -half complex s-plane, $\operatorname{Re}(s) \leq 0$, onto the unit circle $|z| \leq 1$ in the z-plane, we then have the relations

$$
\left.\begin{array}{l}
A=\left(F+I_{n}\right)^{-1}\left(F-I_{n}\right) \\
B=\sqrt{2}\left(F+I_{n}\right)^{-1} G \\
C=\sqrt{2} H\left(F+I_{n}\right)^{-1} \\
D=J-H\left(F+I_{n}\right)^{-1}
\end{array}\right\}
$$

Or, in another form for bilinear transformation.

$$
G(s)=C\left[s I_{n}-A\right]^{-1} B+D \underset{z=\frac{1+s}{1-s}}{\stackrel{s=\frac{z-1}{z+1}}{\longrightarrow}} G(z)=H\left(Z I_{n}-\Phi\right)^{-1} G+J
$$

\section{4..2. Error Bound Analysis of ROM}

If the reduced system has been obtained of the original system, then the modelling error transfer function is well-defined via

$$
E_{r}(s)=\left[G_{o}(s)-G_{r}(s)\right]
$$

The actual infinity norm $\left(H_{\infty}\right)$ error bound in $\boldsymbol{r}^{\text {th }}$ a ROM may be calculated by taking $H_{\infty}$ of $E_{r}(s)$. The actual and theoretical infinity error bounds are given by $\left\|E_{r}(s)\right\|_{\infty}$ and $\leq 2 \sum_{i=r+1}^{n=\text { order of system }} \sigma_{i}$ respectively, Furthermore, the actual amount of error bound is continuously less than or equal to the theoretical amount of error bound. Bound of the system[47][14][2]. Thus

$$
\left\|E_{r}(s)\right\|_{\infty}=\left\|\left[G_{o}(s)-G_{r}(s)\right]\right\|_{\infty} \leq 2 \sum_{i=r+1}^{n} \sigma_{i}
$$

it is also known as a Priori Error bound.

\section{Numerical Experiments}

Example 1: Consider the third-order transfer function of a real-time single-area power system model taken from previous work as [58][59] [60][1][61] with a non-reheated turbine as

In the form of Frequency domain

$$
\frac{250.00}{s^{3}+15.880 s^{2}+42.460 s+106.20}
$$

Expression of Finding the State Space Form

$$
\begin{gathered}
\frac{Y(s)}{U(s)}=\frac{250.00}{s^{3}+15.880 s^{2}+42.460 s+106.20} \\
{\left[s^{3}+15.88 s^{2}+42.46 s+106.2\right] Y(s)=U(s)}
\end{gathered}
$$

Taking Inverse Laplace Transform, we Obtain

$$
\dddot{y}(t)+15.880 \ddot{y}(t)+42.460 \dot{y}(t)+106.20 y(t)=u(t)
$$

Let the chosen state variable be 


$$
\begin{gathered}
x_{1}(t)=y(t) \\
x_{2}(t)=\dot{y}(t)=\dot{x}_{1}(t) \text { and } \\
x_{3}(t)=\ddot{y}(t)=\dot{x}_{2}(t)
\end{gathered}
$$

Substituting equation (33) in equation (31), we obtain

$$
x_{3}(t)=-106.20 x_{1}(t)-42.460 x_{2}(t)-15.880 x_{3}(t)+250 u(t)
$$

Representing equation (33) and (35) in matrix form, we obtain the state equation as

In the form of time domain

$$
\begin{gathered}
{\left[\begin{array}{l}
\dot{x}_{1}(t) \\
\dot{x}_{2}(t) \\
\dot{x}_{3}(t)
\end{array}\right]=\left[\begin{array}{ccc}
0 & 1 & 0 \\
0 & 0 & 1 \\
-106.20 & -42.460 & -15.880
\end{array}\right]\left[\begin{array}{l}
x_{1}(t) \\
x_{2}(t) \\
x_{3}(t)
\end{array}\right]+\left[\begin{array}{l}
0 \\
0 \\
1
\end{array}\right] u(t)} \\
y(t)=\left[\begin{array}{lll}
250 & 0 & 0
\end{array}\right]\left[\begin{array}{l}
x_{1}(t) \\
x_{2}(t) \\
x_{3}(t)
\end{array}\right]
\end{gathered}
$$

Comparing with the standard state-space equation, we obtain

$$
\left.\begin{array}{rl}
A & =\left[\begin{array}{ccc}
0 & 1 & 0 \\
0 & 0 & 1 \\
-106.20 & -42.460 & -15.880
\end{array}\right], B^{T}=\left[\begin{array}{lll}
0 & 0 & 1
\end{array}\right] \\
C & =\left[\begin{array}{lll}
250 & 0 & 0
\end{array}\right], D=0
\end{array}\right\}
$$

It can be demonstrated that the system can be controllable, observable and state. We get Hankel's unique matrix by calculation.

$$
\sigma=\left[\begin{array}{lll}
2.0694 & 0.9222 & 0.0299
\end{array}\right]
$$

From the matrix $\sigma$, it can be proved that $\sigma_{2}>>\sigma_{3}$ The first-second singular values are important here, and then the third singular values have rapidly decayed to become insignificant. Therefore, the order of reduction has been chosen as $2^{\text {nd }}$ order.

\section{The Steps of reduction algorithm using the BT approach are given below}

Step1: The controllability grammians $\left(G_{c}\right)$ and the observability grammians ( $G_{o}$ ) of the system are calculated as for a stable, the controllability $\mathrm{G}$ grammians ramming of $(\mathrm{A}, \mathrm{B})$ is defined the observability grammians of $(\mathrm{C}, \mathrm{A})$. These grammians can be calculated by solving the Lyapunov equations for controllability grammians $\left(G_{c}\right)[44]$,

$$
\begin{aligned}
& {\left[\begin{array}{ccc}
0 & 1 & 0 \\
0 & 0 & 1 \\
-106.2 & -42.46 & -15.88
\end{array}\right]\left[\begin{array}{lll}
G_{C 11} & G_{C 12} & G_{C 13} \\
G_{C 21} & G_{C 22} & G_{C 23} \\
G_{C 31} & G_{C 32} & G_{C 33}
\end{array}\right]} \\
& +\left[\begin{array}{lll}
{ }_{C}+11 & G_{C 12} & { }_{C 13} \\
{ }_{C 21} & G_{C 22} & { }_{C 23} \\
{ }^{G_{C 31}} & { }^{G_{C 32}} & { }^{G}{ }_{C 33}
\end{array}\right]\left[\begin{array}{ccc}
0 & 0 & -106.2 \\
1 & 0 & -42.46 \\
0 & 1 & -15.88
\end{array}\right]+\left[\begin{array}{l}
0 \\
0 \\
1
\end{array}\right]\left[\begin{array}{lll}
0 & 0 & 1
\end{array}\right]=0
\end{aligned}
$$

$$
A=P P^{T}=\left[\begin{array}{ccc}
P_{11} & 0 & 0 \\
P_{21} & P_{22} & 0 \\
P_{31} & P_{32} & P_{33}
\end{array}\right]\left[\begin{array}{ccc}
P_{11} & P_{21} & P_{31} \\
0 & P_{22} & P_{32} \\
0 & 0 & P_{33}
\end{array}\right]
$$

For observability grammians $\left(G_{o}\right)$

$$
\begin{aligned}
& {\left[\begin{array}{ccc}
0 & 0 & -106.2 \\
1 & 0 & -42.46 \\
0 & 1 & -15.88
\end{array}\right]\left[\begin{array}{lll}
G_{O 11} & { }^{G} O 12 & { }^{G} O 13 \\
{ }^{G} O 21 & { }^{G} O 22 & { }^{G} O 23 \\
G_{O 31} & { }^{G} O 32 & { }^{G} \\
O 33
\end{array}\right]} \\
& +\left[\begin{array}{ccc}
{ }^{G} O 11 & G_{O 12} & { }^{G} O 13 \\
{ }^{G}{ }_{O 21} & G_{O 22} & { }^{G} O 23 \\
{ }^{G} O 31 & G_{O 32} & G_{O 33}
\end{array}\right]\left[\begin{array}{ccc}
0 & 1 & 0 \\
0 & 0 & 1 \\
-106.2 & -42.46 & -15.88
\end{array}\right]+\left[\begin{array}{lll}
250 & 0 & 0
\end{array}\right]\left[\begin{array}{c}
250 \\
0 \\
0
\end{array}\right]=0 \\
& G_{O}=1.0 \mathrm{e}+04 *\left[\begin{array}{ccc}
2.6367 & 0.5546 & 0.0294 \\
0.5546 & 0.2129 & 0.0131 \\
0.0294 & 0.0131 & 0.0008
\end{array}\right]
\end{aligned}
$$

Step 2. The find Cholesky factor of $G_{C}$ and $G_{O}$

$$
\begin{aligned}
& A=P P^{T}=\left[\begin{array}{ccc}
P_{11} & 0 & 0 \\
P_{21} & P_{22} & 0 \\
P_{31} & P_{32} & P_{33}
\end{array}\right]\left[\begin{array}{ccc}
P_{11} & P_{21} & P_{31} \\
0 & P_{22} & P_{32} \\
0 & 0 & P_{33}
\end{array}\right] \\
& P P^{T}=\left[\begin{array}{ccc}
P_{11}^{2} & 0 & 0 \\
P_{21} P_{11} & P_{21}^{2}+P_{22}^{2} & 0 \\
P_{31} P_{11} & P_{31} P_{21}+P_{32} P_{22} & P_{31}^{2}+P_{32}^{2}+P_{33}^{2}
\end{array}\right]
\end{aligned}
$$

We obtain the following

$$
P=\left[\begin{array}{ccc}
\sqrt{A_{11}} & 0 & 0 \\
\frac{A_{21}}{P_{11}} & \sqrt{A_{22}-P_{21}^{2}} & 0 \\
\frac{A_{31}}{P_{11}} & \frac{\left(A_{32}-P_{31} P_{21}\right)}{P_{22}} & \sqrt{A_{33}-P_{31}^{2}-P_{32}^{2}}
\end{array}\right]
$$

and therefore, the following formulas for the entries of $\mathrm{L}$

$$
P_{j j}=\sqrt{A_{j j}-\sum_{k=1}^{j-1} P_{j, k}^{2}}
$$

$$
P_{i j}=\frac{1}{P_{j, j}}\left[A_{i j}-\sum_{k=1}^{j-1} P_{i, k} P_{j, k}\right] \text { for } i>j
$$

So, we obtained

$$
\begin{gathered}
P_{C}=\left[\begin{array}{ccc}
0.0115 & 0 & 0 \\
0.0000 & 0.0297 & 0.0297 \\
-0.0767 & 0.0000 & 0.1774
\end{array}\right] \text { and } \\
P_{O}=\left[\begin{array}{ccc}
162.3778 & 0 & 0 \\
34.1572 & 31.0264 & 0 \\
1.8122 & 2.2151 & 0.1873
\end{array}\right]
\end{gathered}
$$

Step 3. The singular value decomposition (SVD) $P_{O}{ }^{T} P_{C}$ is obtained using equation (18). 


\section{We have}

$=\underbrace{\left[\begin{array}{ccc}-0.9733 & 0.2292 & 0.0073 \\ -0.2293 & -0.9731 & -0.0205 \\ 0.0024 & -0.0216 & 0.9998\end{array}\right]}_{U} \underbrace{\left[\begin{array}{ccc}2.0694 & 0 & 0 \\ 0 & 0.9222 & 0 \\ 0 & 0 & 0.0299\end{array}\right]}_{\Sigma} \underbrace{\left[\begin{array}{ccc}-0.7920 & 0.6082 & 0.0541 \\ -0.5787 & -0.7194 & -0.3842 \\ -0.1948 & -0.3356 & 0.9217\end{array}\right]}$

$$
=S V D(\underbrace{\left[\begin{array}{ccc}
0.0115 & 0 & 0 \\
0.0000 & 0.0297 & 0.0297 \\
-0.0767 & 0.0000 & 0.1774
\end{array}\right]}_{P_{O}^{T}} \underbrace{\left[\begin{array}{ccc}
162.3778 & 34.1572 & 1.8122 \\
0 & 31.0264 & 2.2151 \\
0 & 0 & 0.1873
\end{array}\right]}_{P_{C}})
$$$$
=S V D(\underbrace{\left[\begin{array}{ccc}
1.7238 & 1.0134 & 0.3216 \\
-0.1699 & 0.9205 & 0.3931 \\
-0.0144 & -0.0000 & 0.0332
\end{array}\right]}_{P_{O}^{T} P_{C}})
$$

Where, $\mathrm{U}$ and $\mathrm{V}$ are right and left vectors, known as orthogonal columns matrix. By using a non-singular matrix W (transformation) the model can be transformed into a balancing model with help of transformation matrix.

Step 4. Compute $\sum^{-\frac{1}{2}}=\operatorname{diag}\left(\frac{1}{\sqrt{\sigma_{1}}}, \frac{1}{\sqrt{\sigma_{2}}}, \cdots, \frac{1}{\sqrt{\sigma_{n}}}\right)$ where $\sum=\operatorname{diag}\left(\sigma_{1}, \sigma_{1}, \cdots, \sigma_{n}\right)$

Note that $\sigma_{i}, i=1,2, \cdots, n$ are positive numbers

$$
\sum^{-\frac{1}{2}}=\operatorname{diag}\left(\begin{array}{lll}
0.6951 & 1.0413 & 5.7868
\end{array}\right)
$$

From equation (39). It is evident that $\sigma_{2} \gg \sigma_{3}$ to choose the optimal reduced model after truncated $\sigma_{3}$ singular value.

$$
V=\left[\begin{array}{ccc}
-0.7920 & 0.6082 & 0.0541 \\
-0.5787 & -0.7194 & -0.3842 \\
-0.1948 & -0.3356 & 0.9217
\end{array}\right]
$$

Step 5. By using a non-singular matrix $T$, a model can be converted into a balanced model, which can be achieved as follows: the transformation matrix $\mathrm{T}$ is

$$
\begin{aligned}
& T=P_{c} V \sum^{-\frac{1}{2}} \\
& =\underbrace{\left[\begin{array}{ccc}
0.0115 & 0 & 0 \\
0.0000 & 0.0297 & 0.0297 \\
-0.0767 & 0.0000 & 0.1774
\end{array}\right]}_{\sum_{c}^{-\frac{1}{2}}} \underbrace{\left[\begin{array}{ccc}
-0.7920 & 0.6082 & 0.0541 \\
-0.5787 & -0.7194 & -0.3842 \\
-0.1948 & -0.3356 & 0.9217
\end{array}\right]}_{P_{c}} \\
& =\underbrace{\left[\begin{array}{ccc}
0.6951 & 0 & 0 \\
0 & 1.0413 & 0 \\
0 & 0 & 5.7868
\end{array}\right]}
\end{aligned}
$$

$$
T=\left[\begin{array}{ccc}
-0.0063 & 0.0073 & 0.0036 \\
-0.0119 & -0.0222 & -0.0660 \\
0.0182 & -0.1106 & 0.9224
\end{array}\right]
$$

Step 6. Then the system with coefficient matrix

$$
\left(T A T^{-1}, T B, C T^{-1}\right)
$$

Now the system is balanced which is partitioned as:

$$
\begin{aligned}
& G_{B a l}(s)=\left[\begin{array}{c:c}
T A T^{-1} & T B \\
\hline C T^{-1} & D
\end{array}\right] \\
& T A T^{-1}=\underbrace{\left[\begin{array}{ccc}
-0.0063 & 0.0073 & 0.0036 \\
-0.0119 & -0.0222 & -0.0660 \\
0.0182 & -0.1106 & 0.9224
\end{array}\right]}_{T} \underbrace{\left[\begin{array}{ccc}
0 & 1 & 0 \\
0 & 0 & 1 \\
-106.2 & -42.46 & -15.88
\end{array}\right]}_{A} \\
& *\left[\begin{array}{ccc}
-109.8679 & -28.0578 & -1.5790 \\
38.7612 & -23.2863 & -1.8163 \\
6.8173 & -2.2381 & 0.8976
\end{array}\right] \\
& T A T^{-1}=\left[\begin{array}{ccc}
-0.6024 & 2.5000 & 0.6751 \\
-2.5000 & -1.7885 & -1.8269 \\
0.6751 & 1.8269 & -13.4891
\end{array}\right]=A_{B a l} \\
& T B=\underbrace{\left[\begin{array}{ccc}
-0.0063 & 0.0073 & 0.0036 \\
-0.0119 & -0.0222 & -0.0660 \\
0.0182 & -0.1106 & 0.9224
\end{array}\right]}_{T} \underbrace{\left[\begin{array}{l}
0 \\
0 \\
1
\end{array}\right]}_{B} \\
& T B=\underbrace{\left[\begin{array}{c}
-1.579 \\
-1.816 \\
0.8976
\end{array}\right]}_{T B}=B_{B a l} \\
& C T^{-1}=\underbrace{\left[\begin{array}{lll}
250 & 0 & 0
\end{array}\right]}_{C} \underbrace{\left[\begin{array}{ccc}
-109.8679 & -28.0578 & -1.5790 \\
38.7612 & -23.2863 & -1.8163 \\
6.8173 & -2.2381 & 0.8976
\end{array}\right]}_{T^{-1}} \\
& C T^{-1}=\underbrace{\left[\begin{array}{lll}
-1.579 & 1.816 & 0.8976
\end{array}\right]}_{C T^{-1}}=C_{B a l}
\end{aligned}
$$

And

$$
\begin{aligned}
& D=D_{B a l} \\
& G_{B a l}(s)=\left[\begin{array}{l|l}
A_{B a l} & B_{B a l} \\
\hline C_{B a l} & D_{B a l}
\end{array}\right]==\left[\begin{array}{ccc|c}
-0.6024 & 2.5 & 0.6751 & -1.579 \\
-2.5 & -1.789 & -1.827 & -1.816 \\
0.6751 & 1.827 & -13.49 & 0.8976 \\
\hline-1.579 & 1.816 & 0.8976 & 0
\end{array}\right] \\
& =\underbrace{\left[\begin{array}{cc}
A_{11} & B_{1} \\
C_{1} & D
\end{array}\right]}_{\text {Strong-Subsyustem }}+\underbrace{\left[\begin{array}{cc}
A_{22} & B_{2} \\
C_{2} & 0
\end{array}\right]}_{\text {Weak-Subsystem }} \Leftrightarrow \sum:\left[\begin{array}{cc}
\sum_{1} & 0 \\
0 & \Sigma_{2}
\end{array}\right]
\end{aligned}
$$

Where $A_{11}$ and $\sum_{1}$ are $r \times r(r<n)$ matrices. 


$$
\Sigma_{1}=\underbrace{\left[\begin{array}{cc:c}
-0.6024 & 2.5 & -1.579 \\
-2.5 & -1.789 & -1.816 \\
\hdashline-1.579 & 1.816 & 0
\end{array}\right]}_{\text {Strong-Sulssustem }}, \Sigma_{2}=\underbrace{\left[\begin{array}{cc:c}
-13.489 & 0.8976 \\
\hdashline 0.8976 & 0
\end{array}\right]}_{\text {Weak Subssstem }}
$$

So the states of the balanced system corresponding to singular values $\sigma_{3}$ are truncated and the reduced model $\left(A_{11}, B_{1}, C_{1}\right)$ or $G_{r}(s)$ is obtained as

$$
\begin{gathered}
G_{r}(s)=\frac{-0.8056 s+16.81}{\underbrace{s^{2}+2.391 s+7.327}_{\text {Strong Subssstem }}}=\Sigma_{1}, \\
G_{W S}(s)=\underbrace{\frac{0.8056}{\mathrm{~s}+13.49}}_{\left(\sigma_{3}\right) \text { Weak Subsystem }}=\Sigma_{2}
\end{gathered}
$$

The modified BTM is used to calculate the gain factor K using the equation (14) as obtained.

$$
\begin{gathered}
K=1.02604 \\
\hat{G}_{r}(s)=\frac{-0.8056 s+17.2477}{s^{2}+2.391 s+7.327}
\end{gathered}
$$

We obtained the final reduced-order model after inserting $\mathrm{K}$ into the transfer function $G_{r}(s)$ of $\left(A_{11}, B_{1}, C_{1}\right)$ to gain $\hat{G}_{r}(s)$, making the minimal state -space Realization $\hat{G}_{r}(s)$ to gain $\left(\hat{A}_{11}, \hat{B}_{1}, \hat{C}_{1}\right)$ and

\begin{tabular}{|c|c|c|}
\hline MOR Approaches & ROM & $\boldsymbol{H}_{\infty}$ Norm \\
\hline Proposed Approach & $\frac{-0.8056 s+17.2477}{s^{2}+2.391 s+7.327}$ & 0.013490 \\
\hline Balanced Truncation [57] & $\frac{-0.8056 s+16.810}{s^{2}+2.391 s+7.3270}$ & 0.05979408 \\
\hline Enhanced Balanced Realization [62] & $\frac{-1.26750 s+17.24801}{s^{2}+2.3910 s+7.327}$ & 0.263998061 \\
\hline $\begin{array}{c}\text { Balanced Realization (BR) Method and Factor } \\
\text { Division Method (FDM) [63] }\end{array}$ & $\frac{-1.26720 s+17.24860}{s^{2}+2.3901 s+7.327}$ & 0.263869610 \\
\hline Improved Routh Stability Method [64] & $\frac{250.00}{15.880 s^{2}+35.772 s+106.200}$ & 0.24653080 \\
\hline $\begin{array}{l}\text { Modified Routh Approximation[64], Routh } \\
\text { Approximation and Factor Division [51] }\end{array}$ & $\frac{24.0220 s+8.6880}{s^{2}+13.3799 s+7.9399}$ & 3.21066260 \\
\hline Singular Perturbation Approximation [65][66] & $\frac{15.740}{s^{2}+2.674 s+6.880}$ & 0.48349860 \\
\hline $\begin{array}{l}\text { Stability Equation Method [63]and Pade } \\
\text { Approximation Method[67] }\end{array}$ & $\frac{-0.00460 s+249.995}{15.880 s^{2}+42.460 s+106.2}$ & 0.47710100 \\
\hline Pade Approximation [1] & $\frac{-1.19100 s+18.9200}{s^{2}+2.7080 s+8.0403}$ & 0.12595607 \\
\hline Routh approximation [1] & $\frac{18.680}{s^{2}+3.173 s+7.94}$ & 0.64210960 \\
\hline $\begin{array}{l}\text { Routh Stability method and Factor Division Method } \\
\qquad 68]\end{array}$ & $\frac{39.36600 s+3344.701}{35.80 s^{2}+584.80 s+1420.80}$ & 1.90855030 \\
\hline Differential Method[69] & $\frac{250.00}{5.29300 s^{2}+28.3007 s+106.200}$ & 2.223737203 \\
\hline Routh- Pade Approximation [70] & $\frac{24.0220+8.6880}{s^{2}+13.3790 s+7.939}$ & 3.210662604 \\
\hline Factor Division Method [71] & $\frac{18.8170}{s^{2}+2.5940 s+7.9940}$ & 0.534206074 \\
\hline Routh Stability Method and Pade Approximation[72] & $\frac{-15.748 s+249.9950}{15.88 s^{2}+35.772 s+106.20}$ & 0.440232056 \\
\hline $\begin{array}{l}\text { SEM [73], FDM and Stability Method[22][49] } \\
\text { Truncation Method[74], }\end{array}$ & $\frac{250.00}{15.88 s^{2}+42.467 s+106.200}$ & 0.477107207 \\
\hline Pade- Approximation and Differentiation Method[75] & $\frac{-33.3210 s+249.9950}{2.2930 s^{2}+28.3070 s+106.20}$ & 1.815180029 \\
\hline Routh Stability Method[76] & $\frac{250}{15.88 s^{2}+35.772 s+106.20}$ & 0.246530806 \\
\hline
\end{tabular}
resulting from the order of $\left(\hat{A}_{11}, \hat{B}_{1}, \hat{C}_{1}\right)$, we obtained the final reduced-order model $\left(A_{r}, B_{r}, C_{r}\right)$ as follows

$$
\left.\begin{array}{l}
A_{r}=\left[\begin{array}{cc}
-2.391 & -3.663 \\
2 & 0
\end{array}\right] \\
B_{r}=\left[\begin{array}{l}
4 \\
0
\end{array}\right], C_{r}=\left[\begin{array}{ll}
-0.2064 & 2.156
\end{array}\right], D=0
\end{array}\right\}=R O M
$$

Table 1. A Comparison of the proposed method with Other Existing MOR Method 


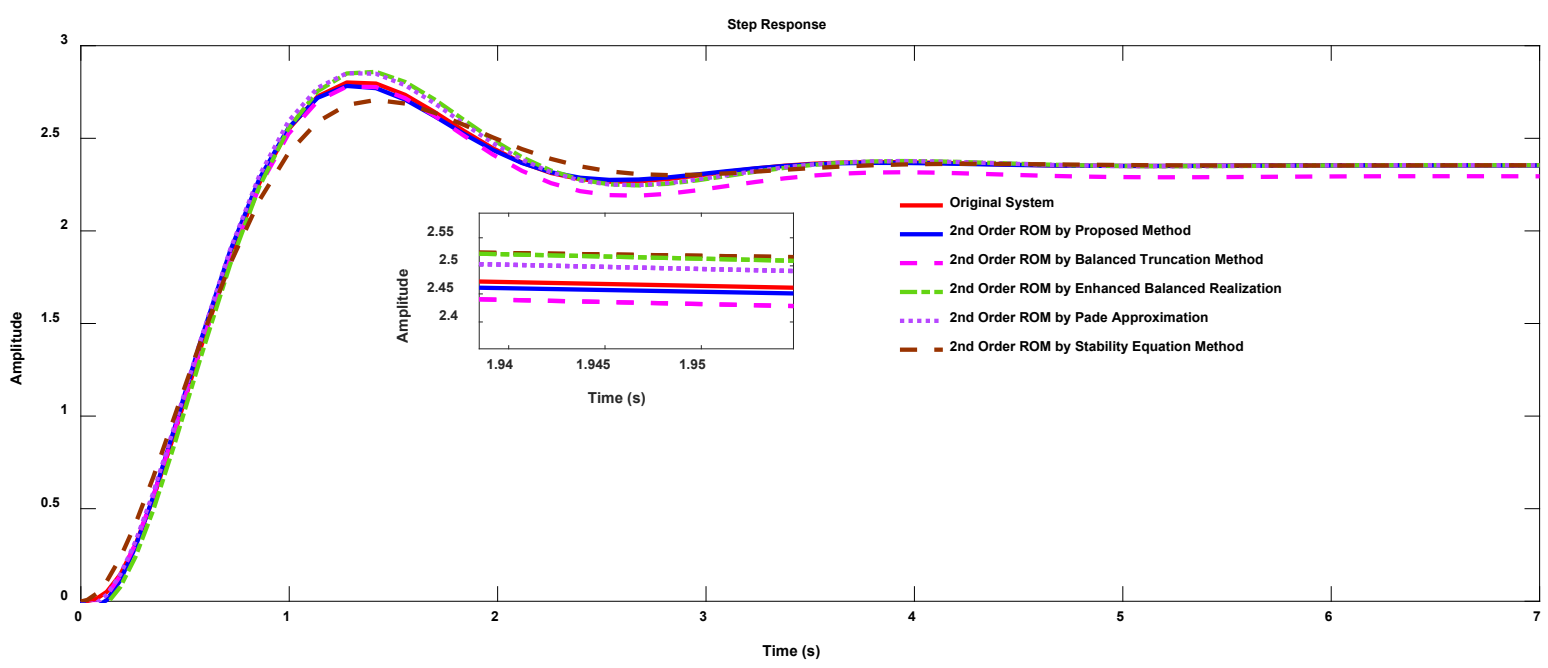

Figure 1. Step Response of The ROM with Original Order System of Continuous System

The step response of the original system, ROM found by a proposed method based upon modified and other methods existing in the literature review are depicted in Fig 1. It has been seen that the ROM so obtained is its closed approximation.

Example 2. We are now considering a discrete SISO system. For comparative purposes, we simply used the bilinear transformation mapping to discretize the system in example 1.

$$
\begin{aligned}
A_{D} & =\left[\begin{array}{ccc}
1.907 & -1.118 & 0.3124 \\
1 & 0 & 0 \\
0 & 0.5 & 0
\end{array}\right] ; B_{D}=\left[\begin{array}{c}
0.5 \\
0 \\
0
\end{array}\right] ; \\
C_{D} & =\left[\begin{array}{lll}
0.1603 & 0.06147 & 0.07553
\end{array}\right], D_{D}=[0.01633]
\end{aligned}
$$

Applying transformation $G(z)$ is transformed to $G(s)$ in state-space form as

$$
\begin{aligned}
A_{C} & =\left[\begin{array}{ccc}
10.2 & -11.77 & 2.617 \\
8.377 & -5.771 & -4.812 \\
-7.702 & 18.87 & -23
\end{array}\right] ; B_{C}=\left[\begin{array}{c}
2.385 \\
-1.804 \\
2.949
\end{array}\right] ; \\
C_{C} & =\left[\begin{array}{lll}
0.1603 & 0.06147 & 0.07553
\end{array}\right], D_{C}=[0.01633]
\end{aligned}
$$

Corresponding Transfer function

$$
G_{C}(s)=\frac{0.01633 s^{3}+0.7973 s^{2}+10.42 s+298.9}{s^{3}+18.57 s^{2}+48.87 s+127}
$$

This is then reduced to $2^{\text {nd }}$ order Model using BTM

$$
\begin{aligned}
A_{C r} & =\left[\begin{array}{cc}
-0.6961 & -2.499 \\
2.499 & -1.725
\end{array}\right] ; B_{C r}=\left[\begin{array}{c}
-1.671 \\
1.718
\end{array}\right] ; \\
C_{C r} & =\left[\begin{array}{ll}
-1.671 & -1.718
\end{array}\right], D_{C r}=[0.01633]
\end{aligned}
$$

The corresponding reduced Transfer function

$$
G_{C r}(s)=\frac{0.0163 s^{2}-0.1229 s+17.23}{s^{2}+2.421 s+7.448}
$$

We can calculate the gain factor $\mathrm{K}$, by the Modified BTM scheme

$$
K=1.017377
$$

After introducing $\mathrm{K}$ into the transfer function $G_{C r}(s)$ of $\left(A_{C 11}, B_{C 1}, C_{C 1}, D_{C 1}\right)$ to gain $\hat{G}_{C r}(s)$, making the minimal state -space Realization $\hat{G}_{C r}(s)$ to gain $\left(\hat{A}_{C 11}, \hat{B}_{C 1}, \hat{C}_{C 1}, \widehat{D}_{C 1}\right)$ and resulting from the order of $\left(\hat{A}_{C 11}, \hat{B}_{C 1}, \hat{C}_{C 1}, D_{C 1}\right)$, we obtained the final reduced-order model $\left(A_{r}, B_{r}, C_{r}, D_{r}\right)$ as follows

$$
\begin{aligned}
& A_{r}=\left[\begin{array}{cc}
-2.421 & -3.724 \\
2 & 0
\end{array}\right] ; B_{r}=\left[\begin{array}{l}
4 \\
0
\end{array}\right] ; \\
& C_{r}=\left[\begin{array}{ll}
-0.04061 & 2.176
\end{array}\right] ; D_{r}=[0.01633]
\end{aligned}
$$

Corresponding reduced Transfer function with inserting gain Factor $K=\frac{G_{C}(s)}{G_{C r}(s)}$

$$
G_{r}(s)=\frac{0.0163 s^{2}-0.1229 s+17.53}{s^{2}+2.421 s+7.448}
$$

Retransformation into z-domain leads to a steady-state error which after steady-state correction is given by

$$
\begin{aligned}
A_{D r} & =\left[\begin{array}{cc}
1.722 & -0.7876 \\
1 & 0
\end{array}\right] ; B_{D r}=\left[\begin{array}{c}
0.5 \\
0
\end{array}\right] ; \\
C_{D r} & =\left[\begin{array}{ll}
0.2597 & 0.0417
\end{array}\right] ; D_{D r}=[0.04739]
\end{aligned}
$$

The corresponding discrete-time reduced Transfer function

$$
G_{D r}(z)=\frac{0.04739 z^{2}+0.04825 z+0.05817}{z^{2}-1.722 z+0.7876}
$$




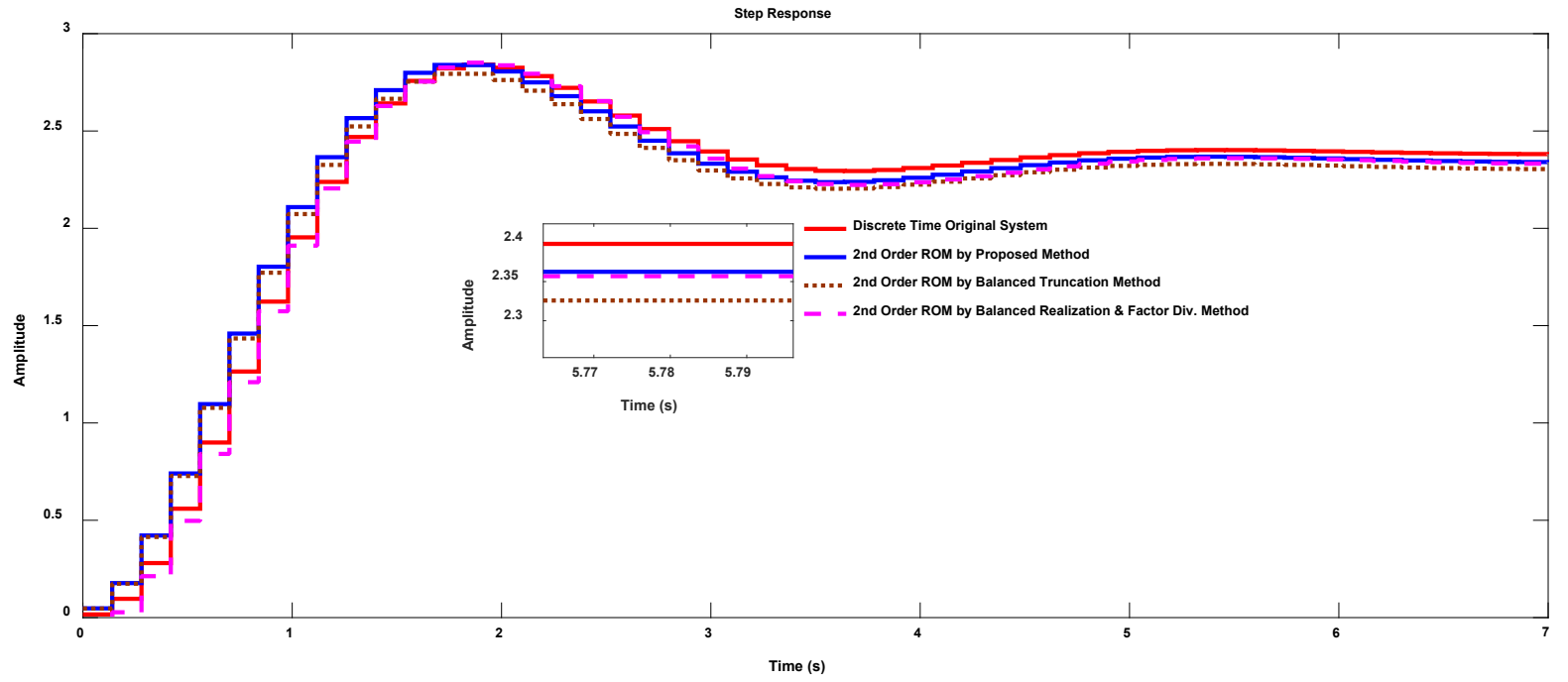

Figure 2. Step Response of The ROM with The Original System of Discrete System

Table 2. A Comparative Summary of Frequency Domain Computations for Single Area Power System

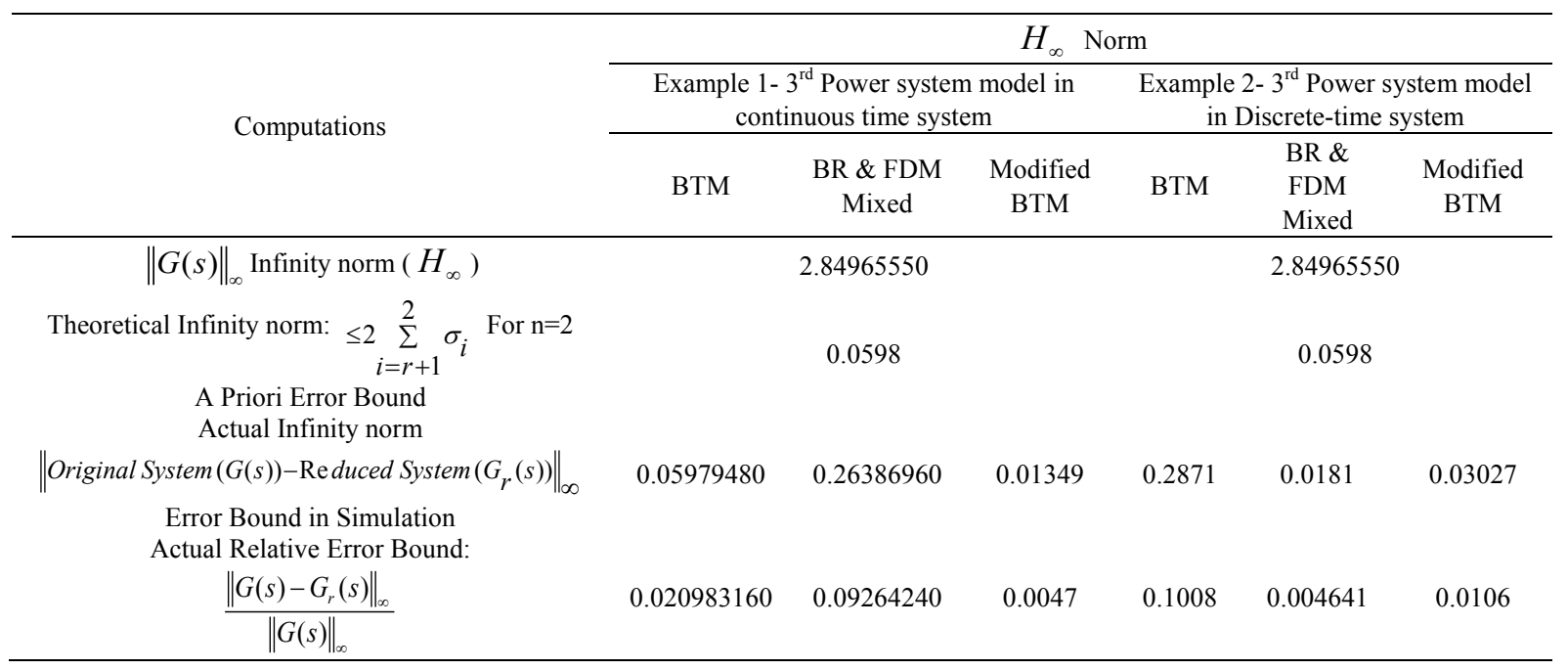

The comparison of step response of the original system, ROM found by the proposed method based upon modified BTM and other methods existing in the literature review are depicted in Fig.2. It has been seen that the ROM so obtained is its close approximation. Further, Measured the accuracy of the proposed method by $H_{\infty}$ Norm value are shown in Table 2. It is observed that the proposed method shows the all error bound value much lesser than the value obtained by other methods available in the literature.

\section{Discussion}

This article presents the step-by-step response of the reduced model and the original system is shown in the figure above. All numerical and Simulation experiments results have been performed on Intel ${ }^{\circledR}$ CoreTM i7-8700 CPU @ $3.20 \mathrm{GHz}$ and 8 GB memory using MATLAB R2019a (Academic Use) at the place of EED, MMMUT,
Gorakhpur. The step responses of the original system and ROM depicted in the figures of both examples are taken from the literature search. This figure shows that the reduced model is very close to the original system. This proposed method's excellence in comparison to the use of the BT method and literature review has been justified through both examples. The $H_{\infty}$ modelling error has been also computed and results are depicted in Tables 1 and Table 2. It is seen to be an excellent precise approximation with a minor error between the original system and ROM. It is observed that the results obtained by the proposed method are far superior. It is clearly stated that the approximation to the Reduced Model of the original system with the lesser error must be closed. It has already been shown that some bilinear mapping not only maintains a balanced structure between a continuous-time system and a discreet time method, but also the Modified BTM structure between a reduced continuous-time order system and a 
reduced discreet time system. The result of continuous-time, stable and balanced system on Modified BTM can easily be extended to a discreet time system.

\section{Conclusion}

A new reduced-order model approach to the reduction of the power system has been proposed in this article. The proposed modified BTM procedure is superior to any conventional method or other mixed methods. This paper has updated the traditional method (BT) to narrow deviations, assuming that dynamic behaviour is maintained. This method drawback has been eliminated by the modified BT of the BTM. The modified BTM is used to effectively display an example of an LTI system and has been easily applied to a discreet time system to support this approach. Also, the time response comparison shows that the ROM calculated using the proposed method provides a close approximation of the HOS. The accuracy, justification and superior performance of the submitted method are also demonstrated by comparing the error standard with the existing literature. The method will become more advantageous when it comes to large-scale continuous/discrete-time systems.

\section{Acknowledgements}

The authors wish to express their appreciation to the Editor and an anonymous referee whose comments and suggestions have improved both the quality and the presentation of the manuscript. I would like to thank my supervisor, Dr. Awadhesh Kumar, for his patience in providing guidance, encouragement and advice throughout my time as a student. I have been extremely lucky to have a supervisor who cared so much about my work and who answered my questions and queries so quickly.

\section{REFERENCES}

[1] S. Saxena and Y. V. Hote, "Load frequency control in power systems via internal model control scheme and model-order reduction," IEEE Trans. Power Syst., 2013, doi: 10.1109/TPWRS.2013.2245349.

[2] S. K. Suman and A. Kumar, "Model Order Reduction of Transmission Line Model," Wseas Trans. Circuits Syst., vol. 19, pp. 62-68, 2020, doi: 10.37394/23201.2020.19.7.

[3] S. K. Suman, A. Narain, and S. Maurya, "RER Based Hybrid Technology for power Generation System:Pollution Free from GHG," SAMRIDDHI A J. Phys. Sci. Eng. Technol., 2018, doi: 10.18090/samriddhi.v10i02.1.

[4] L. L. Grigsby, Power system stability and control. 2017.

[5] C. Ruiz et al., "Dual-Band Reduced-Order Model of an
HVDC Link Embedded into a Power Network for EMT Studies," IEEE Trans. Energy Convers., 2019, doi: 10.1109/TEC.2019.2935892.

[6] E. Kaszkurewicz et al., "Application of balanced realizations for model-order reduction of dynamic power system equivalents," IEEE Trans. Power Deliv., 2016, doi: 10.1109/TPWRD.2016.2551287.

[7] G. Troullinos and J. F. Dorsey, "Application of Balanced Realizations to Power System Equivalents," IEEE Trans. Automat. Contr., 1985, doi: 10.1109/TAC.1985.1103960.

[8] M. Orozco, J. De Jesus Chavez, M. Popov, and P. M. Garcia-Vite, "Dynamic Equivalent Based on Balanced Realizations for Interconnection of Photovoltaic Distributed System," in Proceedings - 2018 IEEE PES Innovative Smart Grid Technologies Conference Europe, ISGT-Europe 2018, 2018, doi: 10.1109/ISGTEurope.2018.8571603.

[9] D. K. Sambariya and O. Sharma, "Routh Approximation: An Approach of Model Order Reduction in SISO and MIMO Systems," Indones. J. Electr. Eng. Comput. Sci., 2016, doi: 10.11591/ijeecs.v2.i3.pp486-500.

[10] A. C. Antoulas, D. C. Sorensen, and S. Gugercin, "A survey of model reduction methods for large-scale systems," 2012.

[11] A. Sikander and R. Prasad, "Soft Computing Approach for Model Order Reduction of Linear Time Invariant Systems," Circuits, Syst. Signal Process., 2015, doi: 10.1007/s00034015-0018-4.

[12] W. H. a Schilders, H. a Van Der Vorst, and J. Rommes, Model Order Reduction: Theory, Research Aspects and Applications. 2008.

[13] K. S. Mohamed, Machine learning for model order reduction. 2018.

[14] D. Boley and B. N. Datta, "Numerical Methods for Linear Control Systems," in Systems and Control in the Twenty-First Century, 1997.

[15] K. E. Willcox and J. Peraire, "Balanced Model Reduction via the Proper Introduction," AIAA J., 2002, doi: $10.2514 / 2.1570$

[16] S. K. Suman and A. Kumar, "Investigation and Reduction of Large-Scale Dynamical Systems,” vol. 18, pp. 175-180, 2019.

[17] W. Schilders, "Introduction to Model Order Reduction," 2008.

[18] A. Dax, "From Eigenvalues to Singular Values: A Review," Adv. Pure Math., 2013, doi: 10.4236/apm.2013.39a2002.

[19] S. K. S, "Approximation of large-scale dynamical systems for Bench-mark Collection," J. Mech. Contin. Math. Sci., 2019, doi: 10.26782/jmcms.2019.06.00016.

[20] S. Gugercin and A. C. Antoulas, "Model reduction of large-scale systems by least squares," Linear Algebra Appl., 2006, doi: 10.1016/j.laa.2004.12.022.

[21] H. Sandberg and A. Rantzer, "Balanced Truncation of Linear Time-Varying Systems," IEEE Trans. Automat. Contr., 2004, doi: 10.1109/TAC.2003.822862.

[22] A. K. Gupta, P. Samuel, and D. Kumar, "A mixed-method 
for order reduction of linear time invariant systems using big bang-big crunch and eigen spectrum algorithm," Int. J. Autom. Control, 2018, doi: 10.1504/ijaac.2019.10018127.

[23] S. K. Suman and A. Kumar, "Model Reduction of Flexible-Missile Control Plant using BST via Schur Method," First Virtual Int. Conf. Latest Adv. Futur. Trends Eng. Sci. Humanit. Manag. Dolphin Coll. Sci. Agric. Chunni Kalan, Punjab, India, pp. 426-433.

[24] X. Cao, M. B. Saltik, and S. Weiland, "Optimal Hankel norm model reduction for discrete-time descriptor systems," $J$. Franklin Inst., 2019, doi: 10.1016/j.jfranklin.2018.11.047.

[25] P. Benner, S. Gugercin, and K. Willcox, "A Survey of Model Reduction Methods for Parametric Systems," SIAM Rev., 2015, doi: 10.1137/130932715.

[26] S. Suman and A. Kumar, "Model Order Reduction by Using Improved Approximation Techniques," Basic Appl. Sci. - Sci. J. King Faisal Univ., vol. 21, no. 2, pp. 80-87, 2020, doi: $10.37575 / \mathrm{b} / \mathrm{eng} / 2246$.

[27] J. Singh, C. B. Vishwakarma, and K. Chattterjee, "Biased reduction method by combining improved modified pole clustering and improved Pade approximations," Appl. Math. Model., 2016, doi: 10.1016/j.apm.2015.07.014.

[28] S. Mukherjee and R. N. Mishra, "Order reduction of linear systems using an error minimization technique," J. Franklin Inst., 1987, doi: 10.1016/0016-0032(87)90037-8.

[29] A. K. Mittal, R. Prasad, and S. P. Sharma, "Reduction of linear dynamic systems using an error minimization technique," J. Inst. Eng. Electr. Eng. Div., 2004.

[30] G. Parmar, S. Mukherjee, and R. Prasad, "System reduction using factor division algorithm and eigen spectrum analysis," Appl. Math. Model., 2007, doi: 10.1016/j.apm.2006.10.004.

[31] S. K. Tiwari and G. Kaur, "Improved Reduced-Order Modeling Using Clustering Method with Dominant Pole Retention," IETE J. Res., 2020, doi: 10.1080/03772063.201 8.1465365 .

[32] S. K. Suman and A. Kumar, "Higher-Order Reduction of Linear System and Design of Controller," Sci. J. King Faisal Univ., vol. 2020, no. 3, pp. 1-16, 2020.

[33] P. Verma, P. K. Juneja, and M. Chaturvedi, "Various Mixed Approaches of Model Order Reduction," in Proceedings 2016 8th International Conference on Computational Intelligence and Communication Networks, CICN 2016, 2017, doi: 10.1109/CICN.2016.138.

[34] B. C. Moore, "Principal Component Analysis in Linear Systems: Controllability, Observability, and Model Reduction," IEEE Trans. Automat. Contr., 1981, doi: 10.1109/TAC.1981.1102568.

[35] K. V. Fernando and H. Nicholson, "On the Structure of Balanced and other Principal Representations of SISO Systems," IEEE Trans. Automat. Contr., 1983, doi: 10.1109/TAC.1983.1103195.

[36] K. V. Fernando and H. Nicholson, "Singular Perturbational Model Reduction of Balanced Systems," IEEE Trans. Automat. Contr., 1982, doi: 10.1109/TAC.1982.1102932.

[37] U. M. Al-Saggaf and G. F. Franklin, "Model Reduction Via Balanced Realizations: An Extension and Frequency
Weighting Techniques," IEEE Trans. Automat. Contr., 1988, doi: $10.1109 / 9.1280$.

[38] Y. Liu and B. D. O. Anderson, "Singular perturbation approximation of balanced systems," Int. J. Control, 1989, doi: 10.1080/00207178908953437.

[39] R. Samar, I. Postlethwaite, and D. W. Gu, "Model reduction with balanced realizations," Int. J. Control, vol. 62, no. 1, pp. 33-64, 1995, doi: 10.1080/00207179508921533.

[40] B. Clapperton, F. Crusca, and M. Aldeen, "Bilinear transformation and generalized singular perturbation model reduction," IEEE Trans. Automat. Contr., 1996, doi: $10.1109 / 9.489281$.

[41] K. Glover, "All optimal Hankel-norm approximations of linear multivariable systems and their L, $\infty$-error bounds $\dagger$," Int. J. Control, 1984, doi: 10.1080/00207178408933239.

[42] D. Škatarić and N. R. Kovačević, "The system order reduction via balancing in view of the method of singular perturbation," FME Trans., 2010.

[43] P. Benner and a Schneider, "Balanced truncation model order reduction for LTI systems with many inputs or outputs," ... theory networks Syst., pp. 1971-1974, 2010.

[44] S. K. Suman, "Reduction of Large-Scale Dynamical Systems by Extended Balanced Singular Perturbation Approximation," vol. 5, no. 5, pp. 939-956, 2020.

[45] Y. Liu and B. D. O. Anderson, "Singular perturbation approximation of balanced systems," in Proceedings of the IEEE Conference on Decision and Control, 1989, doi: 10.1109/cdc.1989.70360.

[46] C. Huang, K. Zhang, X. Dai, and W. Tang, "A modified balanced truncation method and its application to model reduction of power system," in IEEE Power and Energy Society General Meeting, 2013, doi:10.1109/PESMG.2013. 6672263 .

[47] A. C. Antoulas, Approximation of Large-Scale Dynamical Systems. 2011

[48] K. Zhou, G. Salomon, and E. Wu, "Balanced realization and model reduction for unstable systems," Int. J. Robust Nonlinear Control, 1999, doi: 10.1002/(SICI)1099-1239(19 9903)9:3<183::AID-RNC399>3.0.CO;2-E.

[49] A. Sikander and R. Prasad, "Linear time-invariant system reduction using a mixed methods approach," Appl. Math. Model., 2015, doi: 10.1016/j.apm.2015.04.014.

[50] A. C. Antoulas, P. Benner, and L. Feng, "Model reduction by iterative error system approximation," Math. Comput. Model. Dyn. Syst., 2018, doi: 10.1080/13873954.2018.1427116.

[51] A. K. Prajapati and R. Prasad, "Reduced-Order Modelling of LTI Systems by Using Routh Approximation and Factor Division Methods," Circuits, Syst. Signal Process., 2019, doi: 10.1007/s00034-018-1010-6.

[52] F. Ferranti, D. Deschrijver, L. Knockaert, and T. Dhaene, "Data-driven parameterized model order reduction using z-domain multivariate orthonormal vector fitting technique," in Lecture Notes in Electrical Engineering, 2011, doi: 10.1007/978-94-007-0089-5 7.

[53] S. Gugercin, “An iterative SVD-Krylov based method for 
model reduction of large-scale dynamical systems," Linear Algebra Appl., 2008, doi: 10.1016/j.laa.2007.10.041.

[54] M. Imran, A. Ghafoor, and V. Sreeram, "A frequency weighted model order reduction technique and error bounds," Automatica, 2014, doi: 10.1016/j.automatica.2014.10.062.

[55] A. C. Antoulas, "A new result on passivity preserving model reduction," Syst. Control Lett., 2005, doi: 10.1016/j.sysconl e.2004.07.007.

[56] J. Schoukens, R. Relan, and M. Schoukens, "Discrete Time Approximation of Continuous Time Nonlinear State Space Models," IFAC-PapersOnLine, 2017, doi: 10.1016/j.ifacol. 2017.08.1556.

[57] B. C. Moore, "Principal Component Analysis in Linear Systems: Controllability, Observability, and Model Reduction," IEEE Trans. Automat. Contr., 1981, doi: 10.1109/TAC.1981.1102568.

[58] Farid Golnaraghi Benjamin C . Kuo, "Automatic Control Systems Ninth Edition,” in Time, 2010.

[59] B. Sonker, D. Kumar, and P. Samuel, "A modified two-degree of freedom-internal model control configuration for load frequency control of a single area power system," Turkish J. Electr. Eng. Comput. Sci., vol. 25, no. 6, pp. 4624-4635, 2017, doi: 10.3906/elk-1701-225.

[60] W. Tan, "Tuning of PID load frequency controller for power systems," Energy Convers. Manag., 2009, doi: 10.1016/j.enconman.2009.02.024.

[61] W. Tan, "Unified tuning of PID load frequency controller for power systems via IMC," IEEE Trans. Power Syst., 2010, doi: 10.1109/TPWRS.2009.2036463.

[62] A. K. Prajapati and R. Prasad, "Order Reduction in Linear Dynamical Systems by Using Improved Balanced Realization Technique," Circuits, Syst. Signal Process., 2019, doi: 10.1007/s00034-019-01109-x.

[63] A. K. Prajapati and R. Prasad, "Model Order Reduction by Using the Balanced Truncation and Factor Division Methods," IETE J. Res., 2018, doi: $10.1080 / 03772063.2018 .1464971$.

[64] A. K. Prajapati and R. Prasad, "Order Reduction of Linear Dynamic Systems by Improved Routh Approximation Method," IETE J. Res., 2019, doi: 10.1080/03772063.2018. 1452645 .
[65] K. V. Fernando and H. Nicholson, "Singular Perturbational Model Reduction in the Frequency Domain," IEEE Trans. Automat. Contr., 1982, doi: 10.1109/TAC.1982.1103037.

[66] K. Kodra, N. Zhong, and Z. Gajic, "Model order reduction of an islanded microgrid using singular perturbations," in Proceedings of the American Control Conference, 2016, doi: 10.1109/ACC.2016.7525480.

[67] T. C. Chen, C. Y. Chang, and K. W. Han, "Model reduction using the stability-equation method and the Padé approximation method," J. Franklin Inst., 1980, doi: 10.1016/0016-0032(80)90096-4.

[68] N. Singh, R. Prasad, and H. O. Gupta, "Reduction of Linear Dynamic Systems using Routh Hurwitz Array and Factor Division Method," IETE J. Educ., 2006, doi: 10.1080/09747338.2006.11415859.

[69] P. Gutman, C. F. Mannerfelt, and P. Molander, "Contributions to the Model Reduction Problem," IEEE Trans. Automat. Contr., 1982, doi: 10.1109/TAC.1982.110 2930.

[70] R. Prasad, "Pade type model order reduction for multivariable systems using routh approximation," Comput. Electr. Eng., 2000, doi: 10.1016/S0045-7906(00)00002-1.

[71] T. N. Lucas, "FACTOR DIVISION: A USEFUL ALGORITHM IN MODEL REDUCTION.," IEE Proc. D Control Theory Appl., 1983, doi: 10.1049/ip-d.1983.0060.

[72] J. Pal, "Stable reduced-order padé approximants using the routh-hurwitz array," Electron. Lett., 1979, doi: 10.1049/el:19790159.

[73] T. C. Chen, C. Y. Chang, and K. W. Han, "Reduction of Transfer Functions by the Stability-Equation Method," $J$. Franklin Inst., 1979, doi: 10.1016/0016-0032(79)90066-8.

[74] Y. Smamash, "Truncation method of reduction: a viable alternative," Electron. Lett., 2007, doi: 10.1049/el:1981007

[75] K. K. D, "A New Algorithm for Model Order Reduction of Interval Systems," Bonfring Int. J. Data Min., 2013, doi: 10.9756/BIJDM.10131.

[76] A. S. Rao, S. S. Lamba, and S. V. Rao, "Comments on 'Model Reduction Using the Routh Stability Criterion,"' IEEE Transactions on Automatic Control. 1979, doi: 10.1109/TAC. 1979.1102069 